\title{
Brachytherapy boost and cancer-specific mortality in favorable high-risk versus other high-risk prostate
}

\section{cancer}

\author{
Vinayak Muralidhar, MSc', Michael Xiang, MD, PhD², Peter F. Orio III, DO ${ }^{3}$, Neil E. Martin, MD, MPH ${ }^{3}$, Clair J. Beard, MD², \\ Felix Y. Feng, MD ${ }^{4}$, Karen E. Hoffman, MD, MHSc, MPH5 , Paul L. Nguyen, MD ${ }^{3}$ \\ 'Harvard-MIT Division of Health Sciences and Technology, Harvard Medical School, Boston, MA, ²Department of Radiation Oncology, \\ Stanford School of Medicine, Stanford, CA, ${ }^{3}$ Department of Radiation Oncology, Dana-Farber Cancer Institute and Brigham and Women's \\ Hospital, Boston, MA, “Department of Radiation Oncology, University of Michigan, Ann Arbor, MI, ${ }^{4}$ Department of Radiation Oncology, MD \\ Anderson Cancer Center, Houston, USA
}

\begin{abstract}
Purpose: Recent retrospective data suggest that brachytherapy (BT) boost may confer a cancer-specific survival benefit in radiation-managed high-risk prostate cancer. We sought to determine whether this survival benefit would extend to the recently defined favorable high-risk subgroup of prostate cancer patients (T1c, Gleason $4+4=8, \mathrm{PSA}<10 \mathrm{ng} / \mathrm{ml}$ or T1c, Gleason 6, PSA > $20 \mathrm{ng} / \mathrm{ml}$ ).

Material and methods: We identified 45,078 patients in the Surveillance, Epidemiology, and End Results database with cT1c-T3aN0M0 intermediate- to high-risk prostate cancer diagnosed 2004-2011 treated with external beam radiation therapy (EBRT) only or EBRT plus BT. We used multivariable competing risks regression to determine differences in the rate of prostate cancer-specific mortality (PCSM) after EBRT + BT or EBRT alone in patients with intermediate-risk, favorable high-risk, or other high-risk disease after adjusting for demographic and clinical factors.

Results: EBRT + BT was not associated with an improvement in 5-year PCSM compared to EBRT alone among patients with favorable high-risk disease (1.6\% vs. $1.8 \%$; adjusted hazard ratio [AHR]: 0.56; 95\% confidence interval [CI]: $0.21-1.52, p=0.258)$, and intermediate-risk disease (0.8\% vs. $1.0 \%$, AHR: $0.83,95 \%$ CI: 0.59-1.16, $p=0.270)$. Others with high-risk disease had significantly lower 5-year PCSM when treated with EBRT + BT compared with EBRT alone (3.9\% vs. $5.3 \%$; AHR: 0.73 ; 95\% CI: $0.55-0.95 ; p=0.022$ ).

Conclusions: Brachytherapy boost is associated with a decreased rate of PCSM in some men with high-risk prostate cancer but not among patients with favorable high-risk disease. Our results suggest that the recently-defined "favorable high-risk" category may be used to personalize therapy for men with high-risk disease.
\end{abstract}

J Contemp Brachytherapy 2016; 8, 1: 1-6 DOI: $10.5114 / \mathrm{jcb} .2016 .58080$

Key words: brachytherapy, favorable high-risk prostate cancer, high-risk prostate cancer, risk stratification.

\section{Purpose}

ASCENDE-RT ("Androgen Suppression Combined with Elective Nodal and Dose Escalated Radiation Therapy") is a randomized trial testing the role of brachytherapy (BT) boost compared to external beam radiation therapy (EBRT) boost after whole pelvis EBRT for intermediate- to high-risk prostate cancer. The trial recently reported improved biochemical outcomes in the EBRT + BT arm [1]. However, given limited follow-up to date, it is unclear whether this improvement in biochemical recurrence-free survival (bRFS) may translate to a mortality benefit for patients treated with a brachytherapy boost. Similarly, two smaller single-center studies found a bRFS benefit with the addition a BT boost in a similar patient population; in both cases, the secondary endpoint of overall survival was not improved [2,3]. While National Comprehensive Cancer Network (NCCN) guidelines began including EBRT + BT as a treatment option for intermediate-risk disease starting in 2002 [4], the first randomized study to support this practice was published in 2005, and may have supported the use of a BT boost before the preliminary ASCENDE-RT results were recently reported. Recent retrospective data suggests that the benefit of BT boost may be limited to patients with high-risk disease [5], whereas intermediate-risk patients appear to have similar cancer-specific survival whether they receive $\mathrm{EBRT}+\mathrm{BT}$ or EBRT alone.

\footnotetext{
Address for correspondence: Dr. Paul L. Nguyen, MD, Department of Radiation Oncology, Dana-Farber Received: 02.11 .2015 Cancer Institute and Brigham and Women's Hospital, Boston, MA, 75 Francis St., Boston, MA 02115, USA, Accepted: 01.02 .2016 phone: +1-617-732-7936, fax: +1-617-975-0912, 凶 e-mail: pnguyen@LROC.harvard.edu Published: 26.02.2016
} 
Other work has suggested that high-risk prostate cancer represents a heterogeneous disease with variable outcomes dependent on the number of risk factors $[6,7,8]$. Among patients managed with radiation, we have recently shown that patients with favorable high-risk disease (stage T1c, Gleason $4+4=8$, and prostate-specific antigen $[\mathrm{PSA}]<10 \mathrm{ng} / \mathrm{ml}$ or stage T1c, Gleason 6, and PSA $>20 \mathrm{ng} / \mathrm{ml}$ ) have much better cancer-specific outcomes than others with high-risk disease and similar outcomes as patients with unfavorable intermediate-risk disease. In particular, estimated 8 year prostate cancer-specific mortality (PCSM) for favorable high-risk disease was $2.1 \%$, compared with $7.1 \%$ for other high-risk disease $(p=0.049)$ and $2.5 \%$ for unfavorable intermediate-risk disease $(p=0.710)$. This finding raises the possibility that patients with favorable high-risk disease might be candidates for less aggressive forms of therapy than is typically recommended for high-risk disease.

Based on the possible benefit of BT boost for patients with high-risk disease seen so far in the ASCENDE-RT trial and in retrospective data, we used a large, national cancer database to investigate whether patients with favorable high-risk prostate cancer benefit from EBRT + BT compared to EBRT alone. Given the lack of a survival benefit seen in retrospective data for intermediate-risk disease, and the similarities in outcome between intermediate-risk disease and favorable high-risk disease, we hypothesized that patients with favorable high-risk prostate cancer would not have a survival benefit from EBRT + BT versus EBRT alone.

\section{Material and methods}

\section{Patient population}

The Surveillance, Epidemiology, and End Results (SEER) database is a population-based cancer registry that covers $28 \%$ of the US population and $97 \%$ of incident cancers. The registry collects cancer diagnostic, treatment, and survival data along with patient demographic characteristics [9]. To match the ASCENDE-RT enrollment criteria, we studied 45,078 patients in the SEER database with cT1c-T3aN0M0 prostate cancer diagnosed between 2004-2011 who had intermediate- or high-risk prostate cancer as defined by NCCN, and were managed with EBRT alone or EBRT + BT. Patients were excluded if they had a PSA over $40 \mathrm{ng} / \mathrm{ml}$ (as in ASCENDE-RT) or received surgical treatment of their cancer [10].

Patients were considered to have intermediate-risk disease if they had at least one intermediate-risk factor (T2b-c, Gleason 7, or PSA $10-20 \mathrm{ng} / \mathrm{ml}$ ) with no high-risk features, and patients were considered to have high-risk disease if they had at least one high-risk factor (T3a, Gleason 8-10, or PSA > $20 \mathrm{ng} / \mathrm{ml}$ ) [10]. In addition, we considered patients to fall into the subset of favorable high-risk disease if they had stage T1c disease with Gleason $4+4=8$ and PSA $<10 \mathrm{ng} / \mathrm{ml}$, or stage T1c disease with Gleason 6 and PSA $>20 \mathrm{ng} / \mathrm{ml}$ [11].

Because of recently reported possible inaccuracies in the recording of a minority of PSA values in the SEER database [12,13], we excluded 2,178 out of 47,256 initial patients $(4.6 \%)$ who had values for PSA and PSA interpretation recorded in SEER that were discordant (e.g. PSA $<4.0 \mathrm{ng} / \mathrm{ml}$ recorded as "positive/elevated" or PSA $>4.0 \mathrm{ng} / \mathrm{ml}$ recorded as "negative/normal"). Otherwise, we only included patients who had known values in SEER for clinical and demographic factors, including $\mathrm{T}$ stage, Gleason score, PSA, reason for not undergoing surgery, marital status, age, income level, education level, year of diagnosis, and race.

\section{Statistical analysis}

Statistical analyses were conducted using Stata/ MP 14.0 (StataCorp, College Station, TX, USA). Fine and Gray competing risks regression [14] was used to determine differences in the risk of PCSM between groups of patients after controlling for T stage (T1c vs. T2 vs. T3a), Gleason score (6 vs. 7 vs. $8-10$ ), the logarithm of the PSA, race (white vs. black vs. other), reason for not receiving surgery (not recommended vs. other), marital status (married vs. other), age at diagnosis, year of diagnosis, income quartile, and educational quartile. Median values were compared using the Kruskall-Wallis test, and proportions were compared using the $\chi^{2}$ test or $t$-test, as appropriate.

$P$ values were reported as statistically significant if less than $\alpha=0.05$.

\section{Results}

\section{Baseline patient characteristics}

Patient characteristics for the cohort are shown in Table 1. Within the entire cohort, median follow-up was 3.6 years, with $28 \%$ of patients $(n=12,621)$ followed for at least 5 years. Men with favorable high-risk disease tended to be diagnosed slightly earlier than those with unfavorable intermediate-risk or other high-risk disease. The majority $(76 \%)$ of patients with favorable high-risk disease had T1c, Gleason 8, and PSA $<10 \mathrm{ng} / \mathrm{ml}$ versus a minority (24\%) with T1c, Gleason 6, and PSA 20-40 ng/ ml. Approximately $20 \%$ of patients received EBRT + BT, and patients with favorable high-risk disease were slightly more likely to receive EBRT + BT compared to intermediate-risk or other high-risk patients. Among patients with favorable high-risk disease, $25.4 \%$ of those with T1c, Gleason $4+4=8$ and PSA $<10 \mathrm{ng} / \mathrm{ml}$ and $15.3 \%$ of those with T1c, Gleason 6, and PSA > $20 \mathrm{ng} / \mathrm{ml}$ received brachytherapy.

\section{Brachytherapy boost and prostate cancer-specific mortality by risk group}

Patients with favorable high-risk disease had similar 5 year PCSM whether they received EBRT + BT or EBRT alone ( $1.6 \%$ vs. $1.8 \%$, adjusted hazard ratio [AHR]: 0.56 , $95 \%$ confidence interval $[\mathrm{CI}]: 0.21-1.52, p=0.258)$. In contrast, others with high-risk disease had a significantly reduced rate of PCSM at 5 years if treated with EBRT + BT compared to EBRT alone $(3.9 \%$ vs. $5.3 \%$, AHR: 0.73, 95\% CI: $0.55-0.95, p=0.022$ ). Like those with favorable high-risk disease, patients with intermediate-risk disease did not have significantly improved 5 year PCSM if they 
Table 1. Baseline patient characteristics

\begin{tabular}{|c|c|c|c|c|c|c|c|}
\hline \multirow[t]{2}{*}{ Patient characteristic } & \multicolumn{2}{|c|}{$\begin{array}{c}\text { Intermediate-risk } \\
(n=30,468)\end{array}$} & \multicolumn{2}{|c|}{$\begin{array}{l}\text { Favorable high-risk } \\
\qquad(n=2,785)\end{array}$} & \multicolumn{2}{|c|}{$\begin{array}{l}\text { Other high-risk } \\
(n=11,825)\end{array}$} & \multirow[t]{2}{*}{$p$} \\
\hline & $n$ & $\%$ & $n$ & $\%$ & $n$ & $\%$ & \\
\hline Median follow-up, years (IQR*) & \multicolumn{2}{|c|}{$3.7(1.7-5.4)$} & \multicolumn{2}{|c|}{$3.4(1.5-5.3)$} & \multicolumn{2}{|c|}{$3.5(1.6-5.3)$} & $<0.001$ \\
\hline Year of diagnosis & & & & & & & 0.001 \\
\hline $2004-2007$ & 15,489 & 50.8 & 1,519 & 54.5 & 6,057 & 51.2 & \\
\hline $2008-2011$ & 14,979 & 49.2 & 1,266 & 45.5 & 5,768 & 48.8 & \\
\hline Median patient age (years) (IQR) & \multicolumn{2}{|c|}{$69(63-74)$} & \multicolumn{2}{|c|}{$70(64-75)$} & \multicolumn{2}{|c|}{$71(65-76)$} & $<0.001$ \\
\hline Race & & & & & & & $<0.001$ \\
\hline White & 23,159 & 76.0 & 2,045 & 73.4 & 8,989 & 76.0 & \\
\hline Black & 5,549 & 18.2 & 550 & 19.7 & 1,915 & 16.2 & \\
\hline Other & 1,760 & 5.8 & 190 & 6.8 & 921 & 7.8 & \\
\hline PSA (ng/ml) & & & & & & & $<0.001$ \\
\hline$<10$ & 20,417 & 67.0 & 2,117 & 76.0 & 4,702 & 39.8 & \\
\hline $10-20$ & 10,051 & 33.0 & - & - & 3,373 & 28.5 & \\
\hline$>20$ & - & - & 668 & 24.0 & 3,750 & 31.7 & \\
\hline Median PSA (ng/ml) & & & & & & & $<0.001$ \\
\hline Gleason score & & & & & & & $<0.001$ \\
\hline$\leq 6$ & 5,258 & 17.3 & 668 & 24.0 & 390 & 3.3 & \\
\hline 7 & 25,210 & 82.7 & - & - & 1,294 & 10.9 & \\
\hline $8-10$ & - & - & 2,117 & 76.0 & 9,283 & 78.5 & \\
\hline T stage & & & & & & & $<0.001$ \\
\hline $\mathrm{T} 1$ & 18,781 & 61.6 & 2,785 & 100.0 & 4,520 & 38.2 & \\
\hline $\mathrm{T} 2$ & 11,533 & 37.9 & - & - & 6,441 & 54.5 & \\
\hline $\mathrm{T3}$ & - & - & - & - & 728 & 6.2 & \\
\hline Radiation treatment & & & & & & & $<0.001$ \\
\hline EBRT & 24,041 & 78.9 & 2,144 & 77.0 & 9,656 & 81.7 & \\
\hline $\mathrm{EBRT}+\mathrm{BT}$ & 6,427 & 21.1 & 641 & 23.0 & 2,169 & 18.3 & \\
\hline
\end{tabular}

$E B R T$ - external beam radiation therapy, $B T$ - brachytherapy, IQR - interquartile range

received EBRT + BT compared to EBRT alone (0.8\% vs. 1.0\%, AHR: 0.83, 95\% CI: 0.59-1.16, $p=0.270$ ) (Figure 1).

\section{Discussion}

In this study, we found that treatment with EBRT + BT boost vs. EBRT alone was not associated with an improvement in PCSM among those with favorable high-risk disease (defined as T1c, Gleason $4+4=8$, PSA $<10 \mathrm{ng} / \mathrm{ml}$ or T1c, Gleason 6, PSA $>20 \mathrm{ng} / \mathrm{ml}$ ), while others with high-risk disease experienced $27 \%$ reduction in the adjusted hazard rate of PCSM and an absolute risk reduction of $1.4 \%(5.3 \%$ to $3.9 \%)$ at 5 years. This study adds support to the notion that patients with radiation-managed highrisk disease represent a heterogeneous group. Previous work has suggested that the survival outcomes of favorable high-risk patients are much better than the outcomes of other high-risk patients and similar to the outcomes of intermediate-risk patients [11]. Here, we show that while patients with other high-risk disease might generally benefit from intensification of radiation therapy via brachytherapy boost, the survival benefit may not extend to the subset of patients with favorable high-risk disease.

The recent report of a bRFS benefit with brachytherapy boost from the ASCENDE-RT randomized trial is likely to increase interest in the use of brachytherapy for patients with intermediate- to high-risk disease [1]. While the trial has not matured far enough to show a cancer-specific or overall survival benefit, it is possible that the striking $53 \%$ improvement in bRFS will translate to a statistically significant mortality benefit with longer follow-up. Until longer follow-up is obtained in the ASCENDE-RT trial, retrospective data might be used to guide application of these preliminary results. Similar to the preliminary results of ASCENDE-RT, two smaller randomized trials, with 218 and 51 patients, have previously shown $31 \%$ and $58 \%$ improvements in bRFS, respectively. Neither study showed an improvement in overall survival, although follow-up was relatively short (7.1 and 8.2 years, respectively), and the studies may have been underpowered for overall survival compared to ASCENDE-RT, which enrolled nearly 400 patients 
A

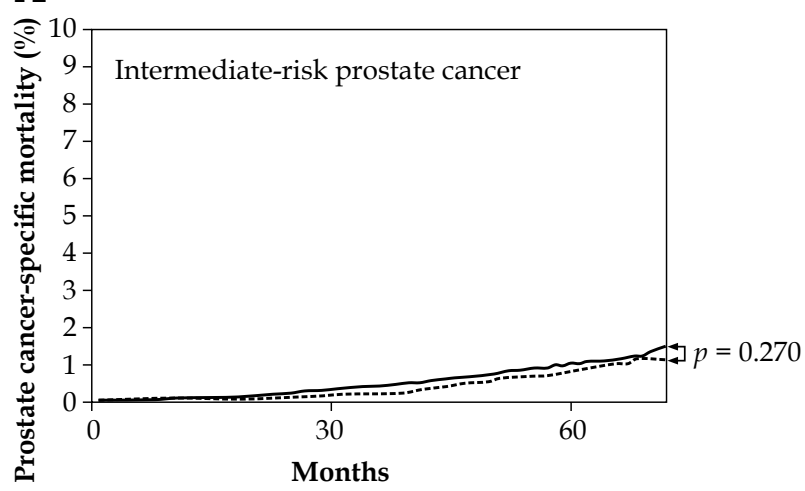

B
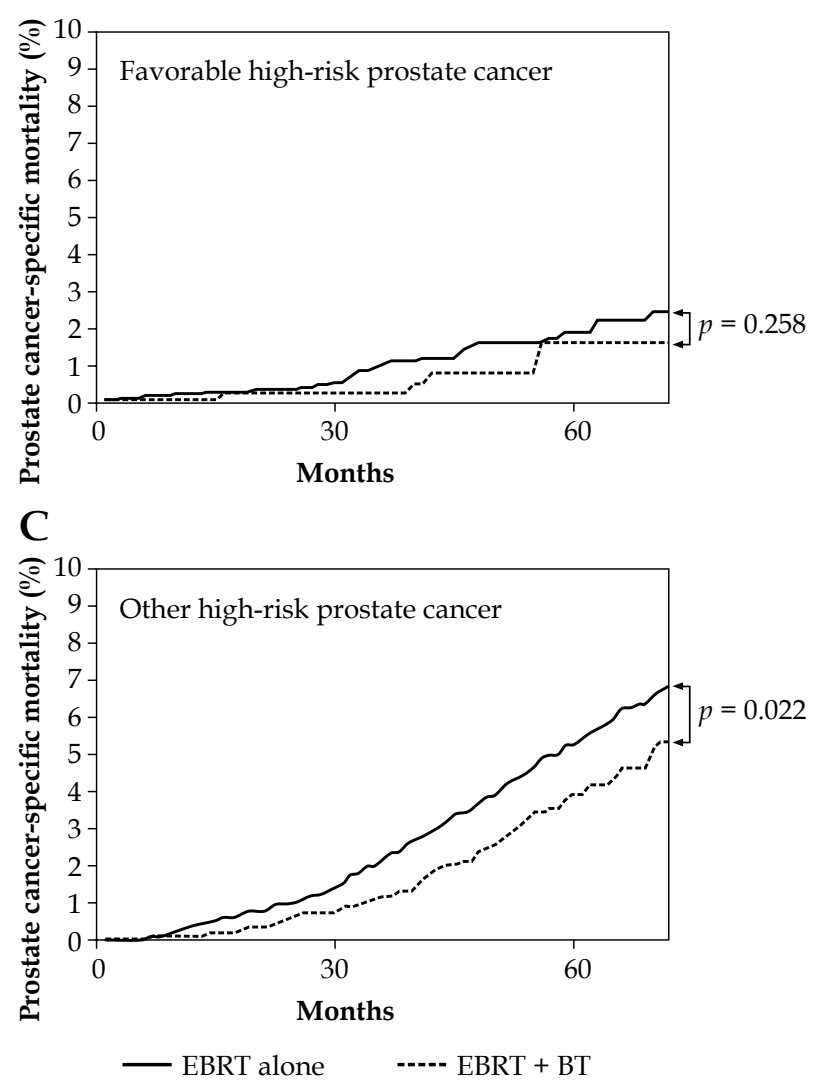

Fig. 1. Prostate cancer-specific mortality among patients with intermediate-risk (A), favorable high-risk (B), or other high-risk $(C)$ prostate cancer treated with external beam radiation therapy $(\mathrm{EBRT})$ or EBRT + brachytherapy (BT)

$[2,3]$. Retrospective data also suggests that the combination of EBRT + BT is superior to EBRT alone, even when very high EBRT doses (86.4 Gy) are used [15]. While only a prospective randomized non-inferiority trial can definitively prove that brachytherapy may be safely omitted in patients with favorable high-risk prostate cancer, these retrospective data may be used until future data from ASCENDE-RT or other studies are available.

Our results are important because it is desirable to identify patients who might not benefit from a BT boost. Brachytherapy for prostate cancer is associated with inher- ent risks, including an increase in the rate of urethral stricture [1], persistent urinary retention requiring further procedures [16,17], or urinary incontinence [16]. In addition, more severe complications such as recto-urethral fistulization or bladder neck contracture may rarely occur [18]. Finally, although not directly related to the procedure, there are inherent risks associated with the use of anesthesia [19]. Although the SEER dataset does not contain information regarding complications from $\mathrm{BT}$, it is possible that the patients in our cohort who received BT are likely to experienced more side effects or toxicity than patients treated with EBRT alone. In the absence of a survival benefit for intermediate-risk or favorable high-risk disease, these patients might be better served by omitting BT from their radiation treatment; although prospective randomized trial data, either as a subgroup analysis of ASCENDE-RT or a separate trial focusing on favorable high-risk disease, will be needed before definitive conclusions can be made regarding the appropriate level of therapy for these patients.

The present study supports the notion that favorable high-risk disease may represent a distinct disease entity within the high-risk group, and that patients with favorable high-risk disease may generally be good candidates for less aggressive therapy than is typically recommended for high-risk disease. Recent data have suggested that patients with favorable high-risk disease, who have clinical T1c (non-palpable) disease and only one other high-risk feature (either Gleason $4+4=8$ and PSA $<10 \mathrm{ng} / \mathrm{ml}$ or Gleason 6 and PSA > $20 \mathrm{ng} / \mathrm{ml}$ ), have significantly better outcomes than others with high-risk disease and similar outcomes as those with unfavorable intermediate-risk disease [11]. Our results showing a benefit to BT boost for patients with other high-risk but not favorable high-risk or intermediate-risk disease, are consistent with the view that favorable high-risk and intermediate-risk prostate cancer likely represent similar levels of risk. Therefore, it is reasonable to consider similar treatment paradigms for these two risk categories. On the other hand, patients with other high-risk disease are likely to represent a subpopulation of men who would benefit most from a higher intensity of therapy, including long-term ADT and BT boost.

Our study should be viewed with respect to its limitations. First, our data are retrospective. Therefore, our results are susceptible to the general limitations of this study design, including selection bias. While we attempted to adjust for potential differences between the EBRT + BT and EBRT groups, some factors such as the use of high-dose-rate (HDR) versus low-dose-rate (LDR) BT, seed type, dose of radiotherapy, quality of radiation therapy, the specifics regarding EBRT or BT technique, receipt of hormone therapy, or bulk of disease (e.g. number of cores positive and percentage of core involvement), are not available in the SEER database. Only a large randomized trial can fully account for all possible sources of selection bias. In addition, the heterogeneity of the practice of BT, which we were not able to measure or adjust for, limits the general applicability of our results.

Second, our risk classification depended on the recorded values of PSA in the SEER database, which may contain a number of errors, possibly due to misplacement of a decimal point [12]. However, preliminary investiga- 
tions by the SEER program have determined that only approximately $5 \%$ of PSA values led to incorrect classification of PSA category among $<10 \mathrm{ng} / \mathrm{ml}, 10-20 \mathrm{ng} / \mathrm{ml}$, and $>20 \mathrm{ng} / \mathrm{ml}$. Based on the results of Schymura et al. [20], we attempted to account for these erroneous values by removing patients who were listed as having a positive PSA despite PSA $<4 \mathrm{ng} / \mathrm{ml}$ or who were listed as having a negative PSA despite PSA $>4 \mathrm{ng} / \mathrm{ml}$; this approach identified $4.6 \%$ of patients with possibly incorrect PSA values. Although this approach may not have accounted for all possible errors in PSA values, the relatively low rate of incorrect PSA categorization combined with the likely random nature of these coding errors leads us to believe that these errors are not likely to systematically bias our findings or impact our conclusions.

Third, it is possible that we did not find a benefit to BT boost among favorable high-risk patients due to a lack of power or due to the relatively short median follow-up of 3.6 years. However, the favorable high-risk subgroup had a large number $(n=2,785)$ of patients. While a low event rate may still lead to underpowering of the comparison, this low rate of PCSM underscores our conclusions that patients with favorable high-risk disease may not benefit from intensification of therapy in the form of BT boost. Studies with longer follow-up, including ASCENDE-RT, will be required before definitive recommendations for patients with favorable high-risk disease can be made.

Fourth, we did not have access to toxicity or quality of life data. While previous work in other cohorts has suggested an increased rate of toxicity associated with BT for prostate cancer $[1,16,17]$, the SEER database does not contain information about toxicity or quality of life, so we were unable to study differences in these important outcomes between the EBRT and EBRT + BT groups. In addition, it is possible that patients who did not receive BT were more likely to receive hormonal therapy, which is also associated with increased toxicity and side effects $[21,22,23,24,25,26]$. Physicians should continue to weigh the patient-specific risks of toxicity from BT, hormonal therapy, and EBRT when personalizing treatment for patients with unfavorable-risk prostate cancer.

\section{Conclusions}

Patients with favorable high-risk prostate cancer (T1c, Gleason $4+4=8$, and PSA $<10 \mathrm{ng} / \mathrm{ml}$ or T1c, Gleason 6, and PSA $>20 \mathrm{ng} / \mathrm{ml}$ ) do not appear to derive a PCSM benefit from EBRT + BT compared to EBRT alone, while others with high-risk disease were $27 \%$ less likely to die from prostate cancer if they were treated with EBRT + BT. Pending long-term survival data from the ASCENDE-RT trial, these results suggest that personalization of therapy is possible within high-risk prostate cancer, and raise the possibility that adding BT boost may not provide a survival benefit for the favorable high-risk subgroup.

\section{Disclosure}

Dr. Paul L. Nguyen has served as a consultant for Medivation and GenomeDx.

The other authors report no conflict of interest.

\section{References}

1. Morris WJ, Tyldesley S, Pai HH et al. ASCENDE-RT: A multicenter, randomized trial of dose-escalated external beam radiation therapy (EBRT-B) versus low-dose-rate brachytherapy (LDR-B) for men with unfavorable-risk localized prostate cancer. J Clin Oncol 2015; 33 (Suppl 7): abstract 3.

2. Hoskin PJ, Rojas AM, Bownes PJ et al. Randomised trial of external beam radiotherapy alone or combined with highdose-rate brachytherapy boost for localised prostate cancer. Radiother Oncol 2012; 103: 217-222.

3. Sathya JR, Davis IR, Julian JA et al. Randomized trial comparing iridium implant plus external-beam radiation therapy with external-beam radiation therapy alone in node-negative locally advanced cancer of the prostate. J Clin Oncol 2005; 23: 1192-1199.

4. NCCN Clinical Practice Guidelines in Oncology, Prostate Cancer. 2002.

5. Xiang M, Nguyen PL. Significant association of brachytherapy boost with reduced prostate cancer-specific mortality in contemporary patients with localized, unfavorable-risk prostate cancer. Brachytherapy 2015; 14: 773-780.

6. Joniau S, Briganti A, Gontero P et al. Stratification of highrisk prostate cancer into prognostic categories: a European multi-institutional study. Eur Urol 2015; 67: 157-164.

7. Walz J, Joniau S, Chun FK et al. Pathological results and rates of treatment failure in high-risk prostate cancer patients after radical prostatectomy. BJU Int 2011; 107: 765-770.

8. Spahn M, Joniau S, Gontero P et al. Outcome predictors of radical prostatectomy in patients with prostate-specific antigen greater than $20 \mathrm{ng} / \mathrm{ml}$ : a European multi-institutional study of 712 patients. Eur Urol 2010; 58: 1-7; discussion 10-1.

9. Overview of the SEER Program: National Cancer Institute; (3 September 2015). Available from: http://seer.cancer.gov/ about/overview.html.

10. NCCN Clinical Practice Guidelines in Oncology, Prostate Cancer. 2015.

11. Muralidhar V, Chen MH, Reznor G et al. Definition and validation of "favorable high-risk prostate cancer": implications for personalizing treatment of radiation-managed patients. Int J Radiat Oncol Biol Phys 2015; 93: 828-835.

12. Furlow B. US National Cancer Institute investigates PSA coding errors. Lancet Oncol 2015; 16: 614.

13. PSA Values and SEER Data.: Surveillance, Epidemiology, and End Results Program; (updated April 29th, 2015September 1st, 2015). Available from: http:// seer.cancer.gov/data/ psa-values.html.

14. Fine JP, Gray RJ. A Proportional Hazards Model for the Subdistribution of a Competing Risk. J Am Stat Assoc 1999; 94: 496-509.

15. Spratt DE, Zumsteg ZS, Ghadjar P et al. Comparison of highdose (86.4 Gy) IMRT vs combined brachytherapy plus IMRT for intermediate-risk prostate cancer. BJU Int 2014; 114:360-367.

16. Stone NN, Stock RG. Complications following permanent prostate brachytherapy. Eur Urol 2002; 41: 427-433.

17. Benoit RM, Naslund MJ, Cohen JK. Complications after prostate brachytherapy in the Medicare population. Urology 2000; 55: 91-96.

18. Moreira SG, Jr., Seigne JD, Ordorica RC et al. Devastating complications after brachytherapy in the treatment of prostate adenocarcinoma. BJU Int 2004; 93: 31-35.

19. Lim KH, Lu JJ, Wynne CJ et al. A study of complications arising from different methods of anesthesia used in high-doserate brachytherapy for cervical cancer. Am J Clin Oncol 2004; 27: 449-451.

20. Schymura MJ, Sun L, Percy-Laurry A. Prostate cancer collaborative stage data items - their definitions, quality, usage, 
and clinical implications: a review of SEER data for 20042010. Cancer 2014; 120 Suppl 23: 3758-3770.

21. Nguyen PL, Alibhai SM, Basaria S et al. Adverse effects of androgen deprivation therapy and strategies to mitigate them. Eur Urol 2015; 67: 825-836.

22. Efstathiou JA, Bae K, Shipley WU et al. Cardiovascular mortality and duration of androgen deprivation for locally advanced prostate cancer: analysis of RTOG 92-02. Eur Urol 2008; 54: 816-823.

23. Nanda A, Chen MH, Braccioforte MH et al. Hormonal therapy use for prostate cancer and mortality in men with coronary artery disease-induced congestive heart failure or myocardial infarction. JAMA 2009; 302: 866-873.

24. Alibhai SM, Duong-Hua M, Sutradhar R et al. Impact of androgen deprivation therapy on cardiovascular disease and diabetes. J Clin Oncol 2009; 27: 3452-3458.

25. D'Amico AV, Denham JW, Crook J et al. Influence of androgen suppression therapy for prostate cancer on the frequency and timing of fatal myocardial infarctions. J Clin Oncol 2007; 25: $2420-2425$.

26. Lee M, Jim HS, Fishman M et al. Depressive symptomatology in men receiving androgen deprivation therapy for prostate cancer: a controlled comparison. Psychooncology 2015; 24: $472-477$. 